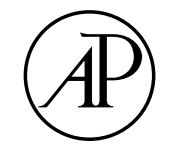

ACADEMIC PRESS

\title{
The role of phonological and orthographic information in lexical selection ${ }^{\text {th }}$
}

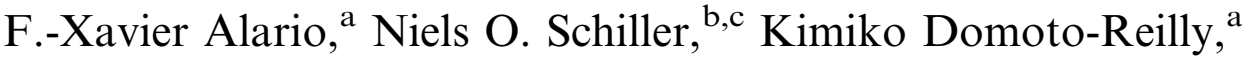 \\ and Alfonso Caramazza ${ }^{\mathrm{a}, *}$ \\ ${ }^{a}$ Harvard University, William James Hall, 33, Kirkland St., Cambridge, MA 02138, USA \\ ${ }^{\mathrm{b}}$ University of Maastricht, Maastricht, The Netherlands \\ ${ }^{\mathrm{c}}$ Max Planck Institute for Psycholinguistics, Nijmegen, The Netherlands
}

Accepted 25 June 2002

\begin{abstract}
We report the performance of two patients with lexico-semantic deficits following left MCA CVA. Both patients produce similar numbers of semantic paraphasias in naming tasks, but presented one crucial difference: grapheme-to-phoneme and phoneme-to-grapheme conversion procedures were available only to one of them. We investigated the impact of this availability on the process of lexical selection during word production. The patient for whom conversion procedures were not operational produced semantic errors in transcoding tasks such as reading and writing to dictation; furthermore, when asked to name a given picture in multiple output modalities - e.g., to say the name of a picture and immediately after to write it down-he produced lexically inconsistent responses. By contrast, the patient for whom conversion procedures were available did not produce semantic errors in transcoding tasks and did not produce lexically inconsistent responses in multiple picture-naming tasks. These observations are interpreted in the context of the summation hypothesis (Hillis \& Caramazza, 1991), according to which the activation of lexical entries for production would be made on the basis of semantic information and, when available, on the basis of form-specific information. The implementation of this hypothesis in models of lexical access is discussed in detail. (c) 2003 Elsevier Science (USA). All rights reserved.
\end{abstract}

\section{Introduction}

Lexical access is the process by which a communicative intention leads to the spoken or written production of words that convey the corresponding message.

\footnotetext{
The authors wish to thank WB and EA for their cheerful cooperation in the studies reported here. We also thank Brad Mahon, Kevin Shapiro, and Adam Szczegielniak for their help in the collection of parts of the data, as well as Rita Capasso and Gabriele Miceli for their comments on a previous version of the manuscript. This work was in part supported by NIH Grant DC 04542 to Alfonso Caramazza. F.-Xavier Alario was supported by a post-doctoral fellowship from the Fyssen foundation. Niels O. Schiller is now supported by the Royal Dutch Academy of Arts and Sciences (KNAW).

* Corresponding author. Fax: 1-617-496-6262.

E-mail address: caram@wjh.harvard.edu (A. Caramazza).
} 
Central to this process is the activation and selection of entries in the mental lexicon, as well as the retrieval of the different properties of the words required for production. According to current models of language production, various stages can be distinguished in the process of lexical access (Caramazza, 1997; Dell, 1986; Garrett, 1975; Levelt, Roelofs, \& Meyer, 1999). These stages can be illustrated by considering the simple situation in which a speaker is asked to name the picture of an object. In this case, the message to be communicated is first conceived during the conceptualization stage, for example by identifying the depicted object and activating its corresponding concept. The activation of information at the conceptualization stage leads to the activation of several lexical entries at the lexicalization stage. At this stage, the word that best denotes the object is selected and its lexical properties are retrieved from the mental lexicon. Finally, in the output stage, the selected word is produced in either its spoken or its written form.

In this study we will concentrate on the lexicalization stage. We present the performance of two patients who suffer from comparable acquired deficits in lexicosemantic processing. The main feature of their performance that will be studied here is their production of semantic errors in tasks involving the production of isolated words-for example, producing "tiger" in response to the picture of a lion. In this paper, we describe the pattern of occurrence of these errors in the oral and written modality, and we discuss the constraints that these data impose on a model of the activation and selection of lexical entries.

The organization of the processes of lexical selection has been mainly documented in studies of speech production (e.g., Nickels, 1997, 2001), although there have also been studies that have compared the oral and written production of words (see references below). One of the important questions addressed in these latter studies is the relationship between the phonological and the orthographic output lexicons: is there an interaction between the retrieval of phonological and orthographic lexical representations during oral and written production? In answer to this question, some early studies proposed that during the production of written output, the message to be produced is first phonologically encoded and then converted to orthography by means of phonology to orthography conversion procedures (Geschwind, 1969; Grashey, 1885; Head, 1926; Hecaen \& Angelergues, 1965; Lichtheim, 1885; Luria, 1966). That is to say, the production of orthography would entirely depend on the previous retrieval of phonological information. However, there is now considerable evidence indicating that such phonological mediation is not required. For example, there have been descriptions of patients who present with significantly impaired oral production and relatively preserved written production (Caramazza \& Hillis, 1990; Lhermitte \& Dérouesné, 1974; Miceli, Benvegnù, Capasso, \& Caramazza, 1997; Rapp, Benzig, \& Caramazza, 1997; Semenza, Cipolitti, \& Denes, 1992; etc.). Moreover, in a number of these cases, the deficit in oral production is likely due to a deficit affecting lexical selection during speech production, as opposed to a deficit affecting more peripheral stages of processing such as articulation (see Rapp et al., 1997, for discussion). This pattern is not expected under the hypothesis that the retrieval of phonology necessarily mediates the retrieval of orthography, as any impairment in the lexical retrieval of phonological information would be visible in the written production.

The conclusion that activation of phonological lexical representations is not required for writing does not necessarily imply that the phonological and the orthographic output lexicons are totally independent. In fact, there have been distinct proposals for a "cross-talk" between phonological and orthographic lexical representations. For example, some authors have postulated that such cross-talk would be implemented in part by direct connections between lexical units in the phonological 
and the orthographic lexicons (Bub, Cancelliere, \& Kertesz, 1985; Coslett, 1991; Funnell, 1983; Sartori, Masterson, \& Job, 1987; Schwartz, Saffran, \& Marin, 1980). These studies report cases of patients who read irregular words of which they have very little understanding, if any. Because comprehension is impaired, this good performance cannot be explained by appealing to a semantic route; because the words are irregular, it also cannot be explained by the application of grapheme to phoneme sublexical conversion procedures. To account for the non-semantic reading of irregular words, a third "lexical" route for reading would then seem to be necessary. According to this view, the activation of a lexical unit in the orthographic lexicon would directly activate the corresponding phonological representation. This would ensure that irregular words are correctly produced, since responses would be based on a lexical-and not sublexical - conversion of orthography to phonology. Furthermore, this process would not require semantic mediation.

However, as noted by Hillis and Caramazza (1991, 1995), postulating such connections is not a necessary requirement to account for this type of performance. These authors highlighted the fact that impairment to the semantic system or to print-to-sound conversion procedures is not always absolute. They proposed that the availability of at least partial semantic information and at least partial phonological information might allow the retrieval of the correct lexical item, without the need for direct links between lexical representations. This "summation" hypothesis postulates that reading of poorly understood irregular words is achieved by the interaction of semantic and phonological information in the output lexicon. It allows a principled re-interpretation of the cases presented earlier in favor of the existence of direct lexical connections, without the need for such connections. Patterson and Hodges (1992) also used this account to explain the performance of reading-impaired patients whose errors in reading irregular words were sensitive to frequency, so that irregular words of low frequency yielded more errors than irregular words of higher frequency. Patterson and Hodges (1992) explained the effect of this lexical factor by assuming that the activation of phonological lexical nodes during reading was accomplished both via the application of grapheme-to-phoneme conversion rules and by the semantic route (for evidence of summation in spelling, see Hillis \& Caramazza, 1991, or Rapp, Epstein, \& Tainturier, in press). Notice that adopting the summation hypothesis as a form of cross-talk between orthographic and phonological lexicons would require a description of the mechanism of summation. That is to say, it would require a specification of how the activation produced by semantics and the activation produced by form-specific information converge on a given lexical entry. We will come back to this issue in Section 6.

Converging evidence for the idea that orthographic and phonological lexical entries are activated on the basis of various sources of information has also been found in studies conducted with "multiple picture-naming" tasks. In a typical trial of this task, participants are presented with a picture and asked to produce successive, temporally close naming responses in the two modalities (oral and written). Notice that here the activation of orthographic (or phonological) information is not directly provided by the stimulus - as in reading or spelling - but is rather a consequence of the visual/semantic processing of the picture by the patient.

When confronted with a double naming task, some patients (e.g., PW described by Rapp et al., 1997; WMA, described by Miceli et al., 1997; MGK described by Beaton, Guest, \& Ved, 1997) produce a significant number of lexically inconsistent responses. For example, in response to the picture of a trumpet, these patients might say "orchestra" and immediately after write "trumpet"; or, in response to the picture of a moustache, they might say "moustache" and then write "beard." Crucially, most if not all of the lexically inconsistent responses involve semantically related lexical 
items, suggesting a deficit at the semantic and/or lexical level of processing. Moreover, all these patients suffered from a deficit of phonology-to-orthography and orthography-to-phonology conversion procedures (as evidenced for example by their inability to write or read non-words). The occurrence of inconsistent responses seems to be tied to the availability of orthography-to-phonology or phonology-to-orthography conversion procedures. Patients that suffer from similar lexico-semantic deficits but for whom the conversion procedures are spared (e.g., patients who make semantic errors in picture naming tasks but who are able to read and write non-words) produce virtually no inconsistent responses. For example, PGE and GIM (Miceli \& Capasso, 1997) produced many semantic errors in picture naming tasks, but their oral and written responses in multiple picture-naming were always lexically similar. Moreover, a pattern consistent with this summation hypothesis has been reported where only one of the conversion procedures is damaged. ECA (Miceli, Capasso, \& Caramazza, 1999) presented a lexico-semantic deficit as well as a deficit of the phonology-to-orthography conversion procedures (e.g., he could barely write non-words, $9.6 \%$ correct) but not of the reverse orthography-to-phonology rules (e.g., he was very good, $86 \%$ correct, at reading non-words). When asked to say and then write the name of a picture, ECA produced a significant number of inconsistent responses. However, in the write-then-say task his responses were virtually always lexically consistent. ${ }^{1}$

In short, the summation hypothesis provides a direct interpretation of the cooccurrence of lexically inconsistent responses in oral and written production with damage to sublexical conversion procedures. The hypothesis explains this pattern of results by postulating that the activation of lexical units in the phonological output lexicon is made on the basis of both semantic information and, when available, phonological information stemming from the application of conversion procedures. Similarly, the activation in the orthographic output lexicon would be made on the basis of semantic information and of orthographic information when available. Briefly, if both processes are available, the activation for a response in one modality can have an impact on the response in the other modality. If, however, the conversion procedures are not available, then lexical selection is carried out independently for each output modality, thereby allowing (in an error-prone system) for lexically inconsistent responses. This interpretation suggests that conversion procedures can have an impact on lexical selection.

In the context of the research we have reviewed, we present the cases of two patients whose performances provide insights into the role of phonological and orthographic information in lexical selection. Following left cerebro vascular accidents (CVAs), each of these patients produces a substantial number of semantic errors in picture-naming tasks, presumably because of an impairment at the level of lexical selection. Interestingly, phonology-to-orthography as well as orthography-tophonology conversion procedures are inoperative in one of the patients but are relatively spared in the other. We took advantage of this difference to investigate the impact of the (un)availability of sublexical conversion procedures on the process of lexical selection during word production. We observed that the patient for whom conversion procedures are not operational produced semantic errors in transcoding tasks such as reading and writing to dictation; furthermore, he produced lexically

\footnotetext{
${ }^{1}$ The absence of lexically inconsistent responses in patients with available conversion procedures could appear to have a trivial explanation. For example, when these conversion procedures are available, the patients could provide their second response simply by reading (or writing) their first response. However, this interpretation seems unlikely, as the same absence of inconsistent responses was observed even when the patients did not have direct access to their first response (for instance, if they were not given the opportunity of seeing what they were writing; Miceli et al., 1999).
} 
inconsistent responses in multiple picture-naming tasks. By contrast, the patient for whom conversion procedures are available did not produce semantic errors in transcoding tasks and did not produce lexically inconsistent responses. These observations are in line with the results discussed previously. They illustrate the impact that the availability of form-specific information can have on the process of lexical selection, an observation that has important consequences for our understanding of lexical access.

In the remainder of this paper, we present a comparative assessement of the performance of the two patients. We then discuss in more detail how to account for our observations, focusing on the hypothesis of a cross-talk between output lexicons. Finally, we evaluate the specific constraints that the inclusion of a mechanism of this kind imposes on models of lexical access.

\section{Case reports}

\subsection{Medical record of $W B$}

WB is a 39-year-old, left-handed male who has completed 11 years of school and works as an auto mechanic. He sustained a left CVA on 3/21/97. A CT scan on 3/24/ 97 revealed a large infarct, involving the entire middle cerebral artery (MCA) territory.

WB lives independently and has been working in a garage for the last one and a half years. The data reported here were collected over a period of two years, during which performance was stable.

\subsection{Screener of $W B$}

WB was administered a battery of screening tests to provide a preliminary assessment of his linguistic abilities. Overall his performance appears to be very impaired.

WB had no problem recognizing letters: he responded correctly in 50 of 51 trials involving upper and lower case letter matching. He performed flawlessly in auditory lexical decision (10/10 correct) and was quite good in visual lexical decision (8/10 correct: he responded "word" both for sarcle and breth). In single word repetition, WB was also quite good: he repeated correctly 202 words out of 260 . Of his errors, $43(74 \%)$ were phonological deviations (e.g., pumpkin $\rightarrow$ "pumpin," basket $\rightarrow$ "bastets") possibly due to a mild dysarthria. The other incorrect responses consisted of 11 morphological errors (all but one were pluralizations), 3 mixed errors (phonological and morphological: basket $\rightarrow$ "bastets"), and 1 semantic error (traffic light$\rightarrow$ "stop").

Sentence comprehension was very poor. WB was $40 \%$ correct in grammaticality judgement (4/10 correct), 56\% correct (9/16 correct) in a sentence picture matching task involving active or passive reversible and irreversible sentences, and $10 \%$ correct (1/10 correct) in sentence completion. WB's speech consists basically of isolated words, primarily nouns. He was not able to read or repeat sentences correctly. When asked to describe a picture (the cookie theft picture, Goodglass, Kaplan, \& Barresi, 2001), WB mainly produced isolated words ("cookies fall down oh really giggle washing down water ripping glasses dripping"). This disfluency was even more pronounced in writing, where his production was limited to "cookie jar water."

A preliminary assessment of his ability to produce words in isolation showed that WB often produced incorrect responses in oral or written naming, as well as in 
transcoding tasks such as writing to dictation and reading. Because the focus of this study is on lexical selection, these tasks are described in more detail in a following section.

\subsection{Medical record of $E A$}

EA is an 88-year-old, right-handed woman who has had two strokes; after her second infarct in October 1999, her family noticed a severe decline in her expressive speech. She first suffered a left MCA CVA in 1996. In October 1999, she slipped and fell, briefly losing consciousness. A CT at that time revealed, in addition to the old left MCA infarct, new right caudate head and body lacune damage. She lives alone, although she does not leave her apartment building without assistance.

\subsection{Screener of $E A$}

EA's was also submitted to a preliminary assessement of her linguistic skills. She has negligible hearing loss, as indicated by her performance in a a same/different test of auditory discrimination (e.g., web-wed) where she was $93 \%$ correct (37/40). She has had surgery on her right eye for cataracts, but her vision is sufficient for reading and daily activities.

The patient had no problem recognizing letters (she was $100 \%$ in a letter matching task). Her comprehension of single words is relatively well preserved: she was $100 \%$ correct in auditory lexical decision $(N=10)$ and $90 \%$ in visual lexical decision ( $N=10$; her errors was on a non-word: "kwine"). Single word repetition was good (17/20 correct, $85 \%$; her errors were mostly phonological such as while $\rightarrow$ wild).

Sentence processing was very poor: she was $50 \%$ (chance, $N=10$ ) in a grammaticality judgement task, $61 \%$ in the sentence-picture matching task (chance $=50 \%$, $N=28)$ and $67 \%$ (4/6 correct) in sentence completion. Her speech was relatively fluent, although not very meaningflul, as shown by her description of the cookietheft picture: "The child is a pull at the base pot here. And I don't oh this is flowin' over the sink. That's a hell of a she's a di-dishwashing a doosh I don't know. What what's the the soo- the soo- over here? (points to window) That's a thing over there. No I don't think think think a cookie jar. Stra- dress (points to water). This thing's two (points to water, boy). Oh must be things to s- see through there (points through window to bushes). Oh. No I can't. Oh uh no I can't t- see anything well over there. No the ash and the dishwashing." This description frequently involves generic terms (e.g., "thing") and does not provide much information about what the picture represents.

As with WB, the preliminary assessement of her naming abilitie showed that EA produced a number semantic errors in naming tasks. These are described in a following section.

\section{Test of semantic abilities}

WB's semantic abilities were assessed with various non-verbal and picture-word matching tasks. As can be seen in Table 1, WB performed very well on the nonverbal tasks from the Birmingham Object Recognition Battery (BORB, Riddoch \& Humphreys, 1992).

WB's performance was further assessed in a picture-word matching task. He was presented with a picture and given a word auditorily. The word was either the name of the picture, a semantic foil, or a phonological foil; his task was to determine 
Table 1

Performance of WB, EA, and control subjects in various picture recognition tasks of the BORB (Birmingham Object Recognition Battery, Riddoch \& Humphreys, 1992)

\begin{tabular}{lllc}
\hline Task & WB & EA & Controls \\
\hline Item match & $30 / 31(97 \%)$ & $31 / 32(97 \%)$ & 30 \\
Associative match & $30 / 30(100 \%)$ & $25 / 30(83 \%)$ & 27.5 \\
Minimal feature view & $24 / 25(96 \%)$ & $23 / 25(92 \%)$ & 23.3 \\
Foreshortened view & $23 / 25(92 \%)$ & $21 / 25(84 \%)$ & 21.6 \\
Object decision & $112 / 126(89 \%)$ & $96 / 128(75 \%)$ & 114.7 \\
Easy & $59 / 64(92 \%)$ & $51 / 64(80 \%)$ & \\
Hard & $53 / 62(85 \%)$ & $45 / 64(70 \%)$ & \\
\hline
\end{tabular}

The controls of the BORB are somewhat older than WB, and somewhat younger than EA. [Item match, match two different pictures of an object against a semantic or visual foil; Associative match, decide which of two pictures most clearly associates with a given picture (as in the Pyramid and Palmtrees test, Howard \& Patterson, 1992); Minimal feature view, match the target with a picture of the same object depicted in such a way that the main identifying features are not visible, against a visual foil; Foreshortened view, match two different views of the same object against a semantic or visual foil; Object decision, decide whether a picture represents a real object or a non-object.]

Table 2

Patients' performance in the picture-word matching task

\begin{tabular}{lll}
\hline Conditions & Correct responses \\
\cline { 2 - 3 } & WB & EA \\
\hline Identical & $99 \%(88 / 89)$ & $98 \%(86 / 88)$ \\
Semantic & $80 \%(69 / 86)$ & $76 \%(66 / 87)$ \\
Phonological & $99 \%(83 / 85)$ & $99 \%(84 / 85)$ \\
All & $92 \%(240 / 260)$ & $91 \%(236 / 260)$ \\
\hline
\end{tabular}

Identical: the word is the name of the picture ("bear"-BEAR). Semantic: the word is semantically related to the picture ("lion"-BEAR). Phonological: the word is phonologically related to the picture ("hair"-BEAR).

whether or not the word corresponded to the picture. The pictures were from the Snodgrass and Vanderwart (1980) set and the foils were names of other pictures in the set. WB's performance was fair overall, although he made a number of errors, mostly accepting semantic foils (see Table 2).

EA's performance on the BORB was equivalent to WB's on the item matching task (31/32, 97\% correct), but on the associative matching task her performance dropped to $83 \%$ correct (25/30; see Table 1). She was also tested on the Snodgrass picture-word matching task. Her performance was very similar to that of WB. (see Table 2).

Overall, both patients performed fairly well in tasks assessing picture/word matching, suggesting that they suffer from comparable, mild damage to their semantic systems.

\section{Sub-lexical and lexical processing tasks}

\subsection{Sub-lexical processing}

The availability of sub-lexical conversion procedures was assessed by asking the patients to read and write 80 non-words. WB performed poorly on both tasks. He was unable to read any of the non-words correctly, although on average his 
responses shared $40 \%$ of their phonemes with the target. In 36 of the trials $(45 \%)$ he responded with lexicalizations (e.g., scark $\rightarrow$ "shark," soat $\rightarrow$ "soap"), and in 19 trials $(24 \%)$ he produced "semantic errors" (he responded with a word that was semantically related to a lexicalization of the non-word; e.g., freet $\rightarrow$ "liberty," presumably by way of "free;" fune $\rightarrow$ "radio," presumably by way of "tune"). The other errors consisted of fragments ( 8 trials, 10\%), perseverations (10 trials, $13 \%$ ), and other types of responses including no-responses ( 7 trials, 9\%). WB was unable to write these same non-words when they were dictated to him: in 30 trials he repeatedly produced the responses "clent" or "cent," irrespective of the stimulus. This response pattern clearly demonstrates that WB had a severe impairment in the application of the rules allowing the conversion of graphemes into phonemes and vice versa. Furthermore, the large number of lexicalizations and "semantic errors" observed in the non-word reading task implicates the use of lexical strategies.

In comparison, EA performed much better with non-words. She was $64 \%$ correct in reading non-words (51/80); all but two of her errors were lexicalizations (e.g., pime $\rightarrow$ prime, sleed $\rightarrow$ sled). On average, her erroneous responses shared $81 \%$ of their phonemes with the target. In written spelling to dictation, her performance dropped to $23 \%$ correct $(18 / 80)$, but most of these errors closely resembled the target (e.g., manch $\rightarrow$ mench, spail $\rightarrow$ speled; on average $73 \%$ of the graphemes in the responses were shared with the targets). The high correspondence between EA's response and the target indicates that her sub-lexical processing remains largely available: since non-words have no support from the semantic system, successful performance depends upon sub-lexical procedures.

\subsection{Lexical processing}

The performance of the patients in tasks requiring the production of single words was assessed in oral and written picture naming, reading, and writing to dictation, using the set of items from Snodgrass and Vanderwart (1980). For each patient, the different tasks were administred on different days.

\subsubsection{Oral picture naming}

In this task, WB responded correctly on 112 trials $(43 \%)$ and EA on 169 trials $(65 \%)$. Table 3 provides a summary of the errors by category in this task. The table distinguishes the trials where the lexical item that was used was inappropriate for the picture-e.g., semantic errors (e.g., leg $\rightarrow$ "foot;" jacket $\rightarrow$ "pants") or lexical substitutions-from the trials where the patients selected the right lexical item but produced it incorrectly_phonological or morphological errors. As can be seen in the table, the distribution of errors is very similar for the two patients, the only major difference being that EA produced very few phonological and morphological errors

Table 3

Error distributions of WB and EA in the oral picture naming task $(N=260)$

\begin{tabular}{llrrrr}
\hline Error type & & \multicolumn{2}{c}{ WB (148 errors) } & \multicolumn{2}{c}{ EA (91 errors) } \\
\hline \multirow{2}{*}{ Wrong lexical item } & Semantic error & 60 & $41 \%$ & 47 & $52 \%$ \\
& Unrelated & 8 & $5 \%$ & 7 & $8 \%$ \\
Correct lexical item & Phon & 28 & $19 \%$ & 1 & $1 \%$ \\
& Morph & 19 & $13 \%$ & 2 & $2 \%$ \\
Other & & 33 & $22 \%$ & 34 & $37 \%$ \\
\hline
\end{tabular}

Sem, semantic; phon, phonological; morph, morphological; the Other category includes errors on compounds,circumlocutions, perseverations, no-responses and unscorable trials. 
as compared to WB. The proportion of semantic errors was slightly higher for EA $(52 \%)$ than for WB $(41 \%)$ but this difference was only marginally significant $\left[\chi^{2}(1)=2.81, p=.09\right]$.

\subsubsection{Written picture naming}

The error distribution for written naming was very similar to that for spoken naming (see Table 4). WB produced 72 correct responses (28\%) and $28 \%$ of his errors were semantic substitutions. In this task, as in most writing tasks to be reported below, WB produced many more perseverations and unscorable responses (e.g., "cclecce") than in oral picture naming. EA produced 163 correct responses (63\%); $45 \%$ of her errors were semantic. Here, EA produced a statistically significant larger $\left[\chi^{2}(1)=8.41, p<.01\right]$.

Thus, these patients present qualitatively similar performance patterns in the picture naming tasks. They both produce a substantial number of semantic errorsEA tending to make more than WB. EA makes very few "form errors" (phonological/orthographic/morphological). WB makes many more perseverations and unscorable errors in the written than in the oral modality.

We now turn to the transcoding tasks: reading and writing to dictation. By contrast to picture naming, there are important differences between the two patients' performance on these tasks.

\subsubsection{Reading}

In reading, WB responded correctly on 131 trials $(50 \%)$. As in picture naming, a substantial proportion of the errors were semantic substitutions (31\%). The large numbers of semantic and unrelated lexical substitutions indicates that WB is employing a lexical strategy in this task. In clear contrast, EA was virtually flawless in the reading task: she only produced 9 errors, among which only one bore a semantic (but also a phonological) relationship with the target (shirt $\rightarrow$ "skirt"). A summary of these data can be found in Table 5 .

\subsubsection{Writing to dictation}

The performance of both patients in writing to dictation paralleled their reading performance (see Table 6). WB performed rather poorly (72 correct responses out of $260,28 \%$ ), and many of his errors were semantic substitutions (60 semantic errors, $32 \%$ ), as well as perseverations and unscorable responses. By contrast, EA made few errors.

The patients' performance in the transcoding tasks was further assessed by asking them to read and to write to dictation another collection of words, the CNLab New Dyslexia Battery (the two tasks were administered on widely separated days). In the

Table 4

Error distributions of WB and EA in the written picture naming task $(N=260)$

\begin{tabular}{llrrrr}
\hline Error type & & \multicolumn{2}{c}{ WB (188 errors) } & \multicolumn{2}{c}{ EA (97 errors) } \\
\hline Wrong lexical item & Semantic error & 53 & $28 \%$ & 46 & $48 \%$ \\
& Unrelated & 13 & $7 \%$ & 5 & $5 \%$ \\
Correct lexical item & Orth & 61 & $31 \%$ & 9 & $9 \%$ \\
& Morph & 3 & $2 \%$ & 1 & $1 \%$ \\
Other & & 58 & $31 \%$ & 36 & $37 \%$ \\
\hline
\end{tabular}

Sem, semantic; orth, orthographic; morph, morphological; the Other category includes errors on compounds, circumlocutions, perseverations, no-responses and unscorable trials. 
Table 5

Error distributions of WB and EA in the reading task $(N=260)$

\begin{tabular}{llrrrr}
\hline Error type & & \multicolumn{2}{l}{ WB (129 errors) } & \multicolumn{2}{l}{ EA (9 errors) } \\
\hline \multirow{2}{*}{ Wrong lexical item } & Semantic error & 40 & $31 \%$ & 1 & $11 \%$ \\
& Visual error & 12 & $9 \%$ & 3 & $33 \%$ \\
& Unrelated & 12 & $9 \%$ & - & - \\
Correct lexical item & Phon & 19 & $15 \%$ & 4 & $44 \%$ \\
& Morph & 12 & $9 \%$ & 1 & $11 \%$ \\
& Phon + morph & 5 & $4 \%$ & - & - \\
Other & & 29 & $22 \%$ & - & \\
\hline
\end{tabular}

Sem, semantic; phon, phonological; morph, morphological; the Other category includes errors on compounds, circumlocutions, perseverations, no-responses and unscorable trials.

Table 6

Error distributions of WB and EA in the writing to dictation task $(N=260)$

\begin{tabular}{llrrrr}
\hline Error type & & \multicolumn{2}{c}{ WB (188 errors) } & \multicolumn{2}{c}{ EA (56 errors) } \\
\hline Wrong lexical item & Semantic error & 60 & $32 \%$ & 1 & $2 \%$ \\
& Auditorily related & 7 & $4 \%$ & 5 & $9 \%$ \\
& Unrelated & 18 & $10 \%$ & 1 & $2 \%$ \\
Correct lexical item & Orth & 37 & $20 \%$ & 31 & $55 \%$ \\
& Morph & 3 & $2 \%$ & 11 & $20 \%$ \\
& Orth + morph & 1 & $1 \%$ & 0 & $0 \%$ \\
Other & & 62 & $33 \%$ & 7 & $12 \%$ \\
\hline
\end{tabular}

Sem, semantic; orth, orthographic; morph, morphological; the Other category includes errors on compounds,circumlocutions, perseverations, no-responses and unscorable trials.

battery, there are five sub-lists of words that were selected to test the effects of various dimensions: concreteness, frequency, part of speech, length, and regularity.

\subsubsection{Reading: CNLab new Dyslexia Battery}

In reading, WB was 30\% correct (104/341). Of his errors, $41(17 \%)$ were semantic (see Table 7). The drop in performance compared to the reading of the Snodgrass and Vanderwart (1980) set can be partly attributed to the introduction of non-picturable nouns and words of other grammatical categories. This interpretation is supported by the fact that WB showed an effect of concreteness [abstract nouns led to more errors than concrete nouns; abstract $=1 / 20$ correct; concrete $=14 / 20$ correct; $\chi^{2}(1)=18, p<.01$ ], and an effect of part of speech [nouns: $11 / 28,39 \%$ correct;

Table 7

Error distributions of WB and EA in the reading task with the CNLab New Dyslexia Battery $(N=341)$

\begin{tabular}{llrrrr}
\hline Error type & & \multicolumn{2}{c}{ WB (237 errors) } & \multicolumn{2}{c}{ EA (16 errors) } \\
\hline \multirow{2}{*}{ Wrong lexical item } & Semantic error & 41 & $17 \%$ & - & - \\
& Visual error & 53 & $22 \%$ & - & - \\
& Unrelated & 35 & $15 \%$ & 2 & $13 \%$ \\
\multirow{2}{*}{ Correct lexical item } & Phon & 9 & $4 \%$ & 11 & $69 \%$ \\
& Morph & 16 & $7 \%$ & 2 & $13 \%$ \\
Other & & 83 & $35 \%$ & 1 & $1 \%$ \\
\hline
\end{tabular}

Sem, semantic; phon, phonological; morph, morphological; the Other category includes errors on compounds, circumlocutions, perseverations, no-responses and unscorable trials. 
adjectives: $7 / 28,25 \%$ correct; verbs: $2 / 28,7 \%$ correct; functors: $0 / 20$ correct; $\left.\chi^{2}(3)=15.2, p<.01\right]$. However, WB showed no clear effect of frequency with this word set [high frequency words: 11/20, 55\% correct; low frequency words: $8 / 20,40 \%$ correct; $\left.\chi^{2}(1)<1\right]$. WB was much better with four letter words $[8 / 15,53 \%$ correct] than with longer words [5 letters: $3 / 15,20 \%$ correct; 6 letters: $4 / 15,27 \%$ correct, 7 letters: 4/15, 27\% correct; 8 letters: $3 / 15,20 \%$ correct] although the overall effect of length was not statistically significant $\left[\chi^{2}(4)=5.53, p=.24\right]$. Consistent with the idea that he was reading and writing words mainly on the basis of lexical processing, WB showed no effect of regularity [irregular words: $15 / 36,42 \%$ correct; regular words: $13 / 36,36 \%$ correct; $\left.\chi^{2}(1)<1\right]$.

EA again produced very few errors in reading, none of which was semantic. She had most difficulty with the regularity sublist, where her errors were almost entirely due to regularizations [irregular words: $31 / 36,86 \%$ correct; regular words: $36 / 36$, $100 \%$ correct; $\left.\chi^{2}(1)=5.37, p=.02\right]$. EA showed no other effect.

\subsubsection{Writing to dictation: CNLab new Dyslexia Battery}

WB performed very poorly in writing this word set to dictation. He responded correctly in 30 trials out of 341 (9\% correct). The majority of his errors were perseverations $(137 / 311,44 \%)$; a substantial number of them were semantic (52/311, $17 \%$ ). Given the very few correct responses given by WB in this task, no assessment of the different effects was carried out.

EA's performance dropped to $77 \%$ correct (263/341). The vast majority of her errors bore a close orthographic resemblance to the target: She produced lexically inappropriate responses in 15 cases (19\% of her errors). EA showed no significant effects of abstractness [abstract nouns: 11/20, 50\% correct; concrete nouns: 14/20, $70 \%$ correct; $\chi^{2}(1)<1$ ], no effect of frequency [high frequency words: $21 / 25,84 \%$ correct; low frequency words: $18 / 25,72 \%$ correct; $\chi^{2}(1)=1.05$, n.s.], or part of speech [adjectives: $20 / 28,71 \%$ correct; functors: $17 / 20,85 \%$ correct; nouns: $24 / 28$, $86 \%$ correct; verbs: 19/28, 68\% correct]. However, she showed a length effect [4 letters, 13/15, 87\% correct; 5 letters: 13/15, 87\% correct; 6 letters: $12 / 15,80 \%$ correct, 7 letters: $11 / 15,73 \%$ correct; 8 letters: $10 / 15,67 \%$ correct], but no effect of regularity [irregular words: $29 / 36,81 \%$ correct; regular words: $31 / 36,86 \%$ correct; $\chi^{2}(1)<1$ ] (see Table 8).

In summary then (see Fig. 1), these two patients produce a substantial number of semantic errors in picture naming tasks. This characteristic, together with the fact that both patients showed slightly impaired performance in the picture recognition and the word-picture matching task, suggests that they suffer from damage to the semantic and/or lexical processing systems. When naming a picture, they could be

Table 8

Error distributions of WB and EA in the writing to dictation task with the CNLab New Dyslexia Battery $(N=341)$

\begin{tabular}{llrrrr}
\hline Error type & & WB (311 errors) & \multicolumn{2}{c}{ EA (78 errors) } \\
\hline \multirow{2}{*}{ Wrong lexical item } & Semantic error & 52 & $17 \%$ & 1 & $1 \%$ \\
& Auditory error & 7 & $2 \%$ & 1 & $1 \%$ \\
& Unrelated & 26 & $8 \%$ & 13 & $17 \%$ \\
\multirow{2}{*}{ Correct lexical item } & Orth & 33 & $11 \%$ & 41 & $53 \%$ \\
& Morph & 7 & $2 \%$ & 15 & $19 \%$ \\
Other & Total & 186 & $60 \%$ & 1 & $1 \%$ \\
\hline
\end{tabular}

Sem, semantic; orth, orthographic; morph, morphological; the Other category includes errors on compounds, circumlocutions, perseverations, no-responses and unscorable trials. 


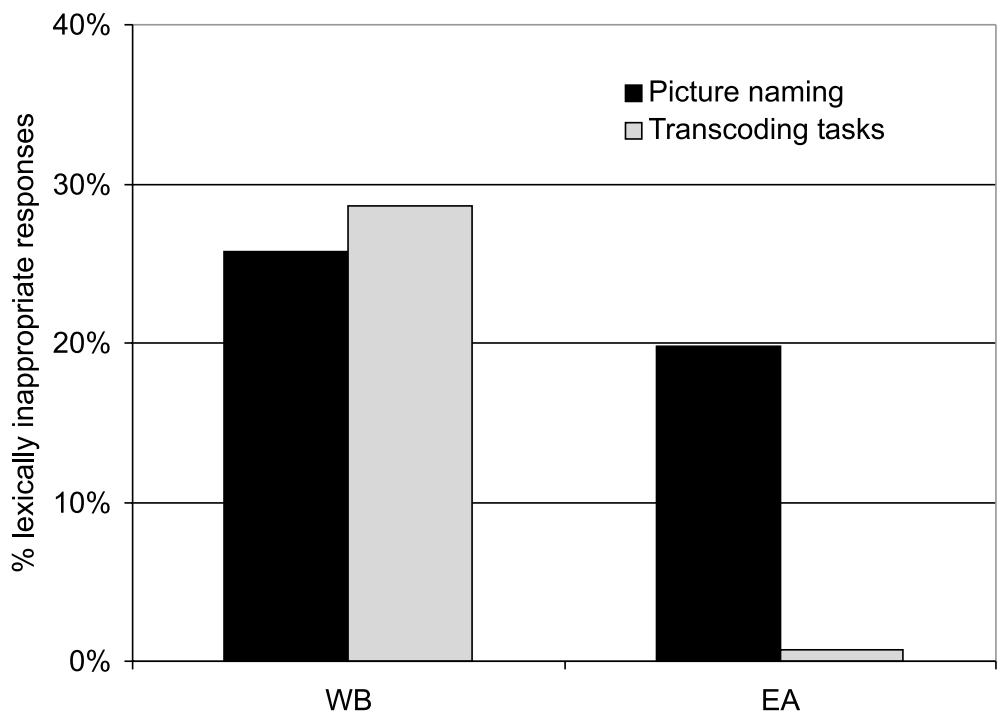

Fig. 1. Overview of the occurrence of lexically inappropriate responses - semantic errors, visual errors, unrelated lexical errors, etc.-for WB and EA in the different single word production tasks. Phonology-toorthography and orthography-to-phonology conversion procedures are impaired for WB, but are available for EA (the data are collapsed over oral and written responses for the Snodgrass \& Vanderwart, 1980, set-for each task type, $N=520$ ).

experiencing difficulties either at the level of identifying the picture or at the level of selecting its name, or both. By contrast, in the transcoding tasks, only WB produced a substantial number of lexical errors. Overall, EA was very good in these tasks: she produced very few lexical mis-selections, and her errors involved minor deviations from the actual phonology or orthography of the correct target (including, e.g., regularizations). These data indicate that when the patient is asked to read or to write words to dictation, the linguistic information encoded by the stimulus can be used to produce the response, provided that the patient has a preserved capacity to use phonology-to-orthography or orthography-to-phonology conversion procedures.

In the context of the studies reviewed in the Introduction, the contrasting performance of these two patients allows predictions about how they will perform lexical selection in a multiple picture-naming task, involving the production of temporally close verbal responses in the two modalities of output-oral and written. The predictions concerning the occurrence of lexically inconsistent responses in relation to the availability of conversion procedures are spelled out and tested in the following section.

\section{Experimental study: Triple naming tasks}

Different predictions can be made for the performance of EA and WB in tasks involving the sequential production of words alternating output modalities. The "summation hypothesis" (Hillis \& Caramazza, 1991) described in the Introduction states that the activation of lexical entries is based on semantic information as well as on form-specific information, when the latter is available. For instance, if phonology-to-orthography or orthography-to-phonology conversion procedures are operational, the production of a word in one modality should lead to the activation of the 
corresponding form-specific representations in the other modality. In the case of EA, the production of a word in one modality (e.g., oral) would produce activation of the corresponding lexical representation in the other modality (e.g., in the orthographic lexicon) via the available conversion procedures. Such pre-activation of the same lexical item would favor its re-use in the second response and will therefore favor the occurrence of lexically consistent sequential responses. In contrast, for WB, whose conversion procedures are not operative, producing a word in one modality should not have major consequences for activation in the other modality of output. Therefore, given the fact that this patient suffers from a lexico-semantic deficit, lexically inconsistent responses in multiple picture-naming tasks can be expected.

These predictions were tested by administering a triple naming task to the patients. In a trial of this task, the patient was asked to name a given picture three times, alternating the modality of output. For example, in one version of the task the patients would say, then write, then say again the name of the picture. In the other version, they would write, then say, then write again the name of the picture. In this last case different paper sheets were used for the two written responses, which were covered right after each response was given. We were interested in determining whether these patients would produce lexically (in)consistent responses in the successive responses of a trial. When lexically inconsistent responses occurred, we further assessed whether the responses would be more consistent within a given modality of output (first and third response) than across modalities (e.g., second and third response).

The same picture set that was used in the previous picture naming tasks was used here (Snodgrass \& Vanderwart, 1980). As the main interest was with the process of lexical selection, we ignored minor phonological, orthographic, and morphological errors, provided that the intended lexical item was recognizable. Moreover, in the assessment of lexical consistency, we only considered those trials in which an "intended lexical item" could be unambiguously identified in all three responses. Trials involving perseverations in any of the three subtasks were also discarded.

\section{1. $W B$}

Consider first the results of the oral-written-oral naming task. In this task, there were 178 scorable trials among the 260 that WB was administered. The error distribution in each of the individual naming trials (Oral 1, Written, and Oral 2) was similar to that observed earlier in single naming (see Table 9).

To evaluate the lexical consistency of the responses, we started by comparing the two initial responses for each modality (Oral 1 and Written). In 122 of the 178 scorable trials (69\%), WB used the same lexical item in the first (oral) and the second

Table 9

Performance of WB in each of the individual tasks of the triple oral-written-oral naming task $(N=260)$

\begin{tabular}{|c|c|c|c|c|c|c|}
\hline & \multicolumn{2}{|c|}{ Oral 1} & \multicolumn{2}{|c|}{ Written } & \multicolumn{2}{|c|}{ Oral 2} \\
\hline & $N$ & $\%$ & $N$ & $\%$ & $N$ & $\%$ \\
\hline Correct & 153 & $59 \%$ & 78 & $30 \%$ & 148 & $57 \%$ \\
\hline All errors & 107 & $41 \%$ & 182 & $70 \%$ & 112 & $43 \%$ \\
\hline Semantic & 49 & $46 \%$ & 42 & $23 \%$ & 51 & $46 \%$ \\
\hline Lexical & 1 & $1 \%$ & 19 & $10 \%$ & 0 & $0 \%$ \\
\hline Phon/orth & 13 & $12 \%$ & 52 & $29 \%$ & 13 & $12 \%$ \\
\hline Morph & 14 & $13 \%$ & 3 & $2 \%$ & 14 & $12 \%$ \\
\hline Other & 30 & $28 \%$ & 66 & $36 \%$ & 34 & $30 \%$ \\
\hline
\end{tabular}


(written) response. Crucial for our purposes, in 56 trials (22\% of all trials, $31 \%$ of the scorable trials) he used different lexical items in his first (oral) and second (written) responses. Among those, 42 trials consisted of a correct oral response followed by an error in the written response and 6 trials consisted of an incorrect response followed by a correct response. Finally, 8 of the inconsistent trials consisted of two different errors. The error distribution can be found in Table 10 .

The performance in this task was further analyzed by evaluating within- and across-modality consistency. For a given trial WB would be consistent within modality if his third response (Oral 2) was the same lexical item as his first response (Oral 1). WB would be consistent across modalities if his third response (Oral 2) was the same lexical item as the one used in the previous response (Written). The data clearly show that WB was more consistent within modality: in the majority of trials his third response was more similar to the first response than to the second response. Consider for example his sequence of responses to a picture of a lion. If he first said "lion" and then wrote "tiger," he was more likely to say "lion" in his third response (42/56 trials, $75 \%)$ than to say "tiger" (6/56 trials, $11 \%)$. In the remaining 8 trials, he produced a different lexical item for each of the responses. A modality-wise summary of the consistency of his responses in the oral-written-oral triple naming task is provided in Table 11.

A similar pattern of performance was observed in the written-oral-written naming task. The distribution of errors for each individual task can be found in Table 12. Again, these distributions were similar to those observed in the simple naming tasks.

WB's responses in this task were similar to the previously reported one. In 127 of the 171 scorable trials $(74 \%)$, WB produced the same lexical item in the first (written) and the second (oral) responses. In 44 of the trials (17\% of all trials, $26 \%$ of scorable trials) he produced different lexical items for these two responses. A summary of the outcomes of the two first responses is provided in Table 10. WB also showed a high degree of within-modality consistency in this task (see Table 11).

To summarize, WB's performances in the oral-written-oral and in the writtenoral-written tasks were very similar. In both tasks he produced lexically inconsistent responses, involving different lexical items in the first and second responses: $31 \%$ of the scorable trials involved lexically inconsistent responses in the oral-written-oral condition and $26 \%$ in the written-oral-written condition. Finally, in most trials the third response involved the lexical item used for the first response rather than that

Table 10

Lexical consistency of WB's responses in the oral-written-oral $(\mathrm{O}-\mathrm{W}-\mathrm{O})$ and the written-oral-written (W-O-W) triple naming tasks

\begin{tabular}{|c|c|c|c|c|c|c|c|}
\hline \multirow[t]{2}{*}{ Type of outcome } & \multicolumn{3}{|c|}{ Examples } & \multicolumn{2}{|c|}{$\mathrm{O}-\mathrm{W}-\mathrm{O}$} & \multicolumn{2}{|c|}{ W-O-W } \\
\hline & Target & First & Second & $N$ & $\%$ & $N$ & $\%$ \\
\hline \multirow{3}{*}{$\begin{array}{l}\text { Consistent } \\
\text { both correct } \\
\text { both incorrect }\end{array}$} & & & & 122 & $69 \%$ & 127 & $74 \%$ \\
\hline & Candle & "candle" & "candle" & & 115 & & 110 \\
\hline & Blouse & "coat" & "coat" & & 7 & & 17 \\
\hline \multirow{4}{*}{$\begin{array}{l}\text { Inconsistent } \\
\text { correct, incorrect } \\
\text { incorrect, correct } \\
\text { incorrect, incorrect }\end{array}$} & & & & 56 & $31 \%$ & 44 & $26 \%$ \\
\hline & Lion & "lion" & "tiger" & & 30 & & 10 \\
\hline & Skirt & "pants" & "skirt" & & 16 & & 25 \\
\hline & Stool & "table" & "chair" & & 10 & & 9 \\
\hline Unscorable & Peacock & "peacock" & "celow" & & 82 & & 89 \\
\hline
\end{tabular}

The two first responses are considered here; for data on the third naming response see Table 11 (First, first response in the trial; Second, second response in the trial, produced in a different modality). 
Table 11

Consistency within and across output modality for WB in the two multiple picture-naming tasks

\begin{tabular}{|c|c|c|c|c|c|c|}
\hline \multirow[t]{2}{*}{ Outcome } & \multicolumn{4}{|l|}{ Examples } & \multirow{2}{*}{$\frac{\mathrm{O}-\mathrm{W}-\mathrm{O}}{N}$} & \multirow{2}{*}{$\frac{\mathrm{W}-\mathrm{O}-\mathrm{W}}{N}$} \\
\hline & Target & First & Second & Third & & \\
\hline \multicolumn{7}{|c|}{ First and second response consistent } \\
\hline third identical & Candle & "candle", & "candle" & "candle" & 118 & 126 \\
\hline third different & Pear & "pear” & "pear" & “apple” & 4 & 1 \\
\hline \multicolumn{7}{|c|}{ First and second response inconsistent } \\
\hline third $=$ first & Lion & "lion" & "tiger" & "lion" & 42 & 41 \\
\hline third $=$ second & Watch & "clock" & "time" & "time" & 6 & 1 \\
\hline third different & Stool & "table" & "chair" & "desk" & 8 & 2 \\
\hline Unscorable & Peacock & "peacock" & "celow" & "peacock" & 82 & 89 \\
\hline
\end{tabular}

Figures in boldface represent those trials where WB was not consistent within modality; these trials are a minority. (First, first response of the trial; Second, second response of the trial; Third, third response of the trial.)

Table 12

Performance of WB in each of the individual tasks of the triple written-oral-written naming task $(N=260)$

\begin{tabular}{|c|c|c|c|c|c|c|}
\hline & \multicolumn{2}{|c|}{ Written 1} & \multicolumn{2}{|c|}{ Oral } & \multicolumn{2}{|c|}{ Written 2} \\
\hline & $N$ & $\%$ & $N$ & $\%$ & $N$ & $\%$ \\
\hline Correct & 82 & $32 \%$ & 152 & $58 \%$ & 74 & $28 \%$ \\
\hline All errors & 178 & $68 \%$ & 108 & $42 \%$ & 186 & $72 \%$ \\
\hline Semantic & 51 & $29 \%$ & 55 & $51 \%$ & 53 & $28 \%$ \\
\hline Lexical & 18 & $10 \%$ & 3 & $3 \%$ & 16 & $9 \%$ \\
\hline Ortho/phonol & 43 & $24 \%$ & 16 & $15 \%$ & 52 & $28 \%$ \\
\hline Morph & 3 & $1 \%$ & 12 & $11 \%$ & 3 & $2 \%$ \\
\hline Other & 63 & $35 \%$ & 22 & $20 \%$ & 62 & $33 \%$ \\
\hline
\end{tabular}

used for the second response. Among the lexically inconsistent trials, WB was consistent within modality in $75 \%$ of the cases in the oral-written-oral condition and in $93 \%$ of the cases in the written-oral-written condition.

\section{2. $E A$}

EA's performance in the each of the tasks involved in the multiple picture-naming task was very similar to her performance in the single naming tasks reported earlier (see Tables 13 and 14).

Interestingly, the lexical consistency pattern observed in WB's performance contrasts sharply with that observed for EA as she was virtually never inconsistent in the multiple picture-naming tasks. In the oral-written-oral version, she always produced lexically consistent responses. In the written-oral-written, she produced lexical inconsistencies on 2 trials (out of 202 scorable trials). For the picture of a football, she wrote "foot ball," said "football" and then wrote "basket ball." For the picture of a cow, she wrote "white cow," then she said "male, female, female calf, moo," and then she wrote "white cow - female." As pointed out in the Introduction, it could be argued that the availability of conversion procedures prevents the occurrence of inconsistent responses because the patients' second response in a trial is provided by reading (or writing) the first response of that trial. For example, in a write-then-say task, the second response would be produced by reading the first response and not by 
Table 13

Performance of EA in each of the individual tasks of the triple oral-written-oral naming task $(N=260)$

\begin{tabular}{|c|c|c|c|c|c|c|}
\hline & \multicolumn{2}{|c|}{ Oral 1} & \multicolumn{2}{|c|}{ Written } & \multicolumn{2}{|c|}{ Oral 2} \\
\hline & $N$ & $\%$ & $N$ & $\%$ & $N$ & $\%$ \\
\hline Correct & 165 & $63 \%$ & 162 & $62 \%$ & 170 & $65 \%$ \\
\hline All errors & 95 & $37 \%$ & 98 & $38 \%$ & 90 & $35 \%$ \\
\hline Semantic & 53 & $56 \%$ & 43 & $44 \%$ & 41 & $46 \%$ \\
\hline Lexical & 2 & $2 \%$ & 4 & $4 \%$ & 5 & $6 \%$ \\
\hline Orth/phon & 1 & $1 \%$ & 12 & $12 \%$ & 4 & $4 \%$ \\
\hline Morph & 5 & $5 \%$ & 3 & $3 \%$ & 6 & $7 \%$ \\
\hline Other & 34 & $36 \%$ & 36 & $37 \%$ & 34 & $37 \%$ \\
\hline
\end{tabular}

Table 14

Performance of EA in each of the individual tasks of the triple written-oral-written naming task $(N=260)$

\begin{tabular}{|c|c|c|c|c|c|c|}
\hline & \multicolumn{2}{|c|}{ Written 1} & \multicolumn{2}{|c|}{ Oral } & \multicolumn{2}{|c|}{ Written 2} \\
\hline & $N$ & $\%$ & $N$ & $\%$ & $N$ & $\%$ \\
\hline Correct & 150 & $58 \%$ & 163 & $63 \%$ & 146 & $56 \%$ \\
\hline All errors & 110 & $42 \%$ & 97 & $37 \%$ & 114 & $44 \%$ \\
\hline Semantic & 41 & $37 \%$ & 41 & $42 \%$ & 42 & $37 \%$ \\
\hline Lexical & 4 & $4 \%$ & 4 & $4 \%$ & 6 & $5 \%$ \\
\hline Orth/phon & 17 & $15 \%$ & 1 & $1 \%$ & 19 & $17 \%$ \\
\hline Morph & 3 & $3 \%$ & 4 & $4 \%$ & 2 & $2 \%$ \\
\hline Other & 45 & $41 \%$ & 47 & $48 \%$ & 45 & $39 \%$ \\
\hline
\end{tabular}

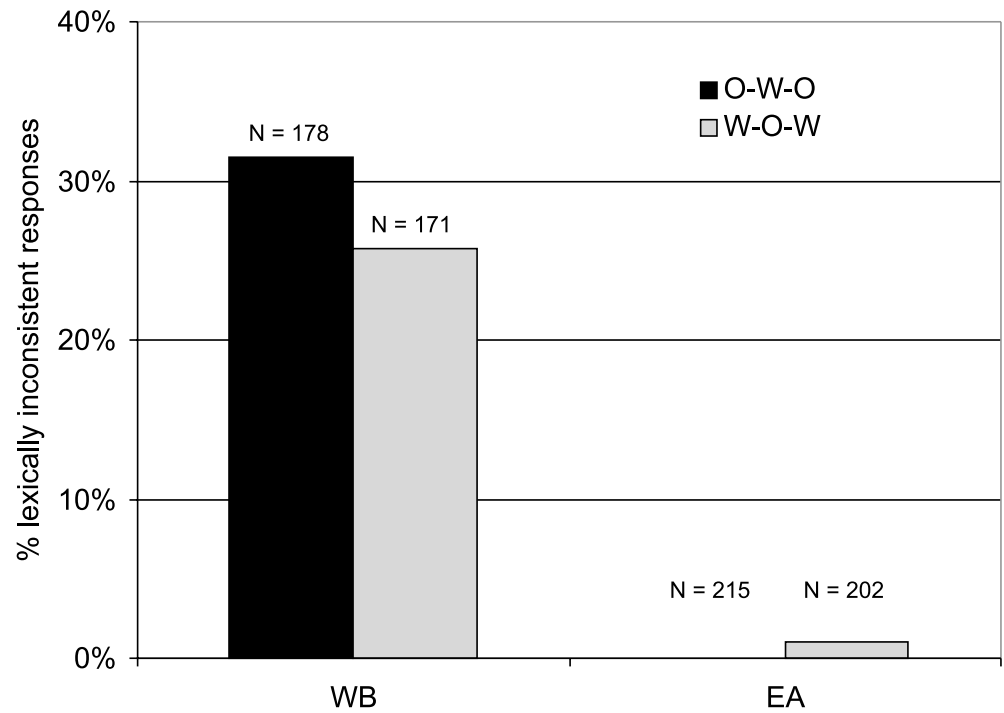

Fig. 2. Overview of the lexical consistency observed in the multiple picture-naming tasks for patients WB and EA. Lexically inconsistent responses are those in which the patient uses different lexical items for a given picture (e.g., lion $\rightarrow$ "lion," then "tiger"). The proportions are calculated based on the total scorable trials for each task (see $N$ in the figure).

processing the picture. However, this seems unlikely, given the fact that not only does EA have bad eyesight, but also that the page she used to provide her first response was removed upon completion of her response (see also Miceli et al., 1999). 
Fig. 2 provides a summary of the data observed in the multiple picture-naming tasks: lexically inconsistent responses were produced by the patient with impaired phonology-to-orthography and orthography-to-phonology conversion procedures (WB), but were virtually never produced by the patient with spared conversion procedures (EA).

\section{General discussion}

In this report, we have documented the performance of two patients-WB and EA-who suffer from a lexico-semantic deficit which affects their performance in language production tasks. When asked to name pictures, regardless of the modality of output, both of them produce a significant number of semantic errors. These errors are most likely due to a deficit in the semantic system and/or a deficit in the process of word retrieval.

Despite this common characteristic, there were important differences between the two patients' performance. WB produced a significant number of semantic errors in transcoding tasks such as reading or writing to dictation. Also, when asked to read non-words, WB made numerous mistakes - in fact, none of his responses was correct, although they tended to resemble the target-and he was virtually unable to write non-words to dictation. This pattern suggests that WB suffered from damage to sublexical conversion procedures-grapheme-to-phoneme or phoneme-to-grapheme conversion - and that he relied mostly on the (deficient) lexical system when transcoding speech or writing. In contrast, EA did not produce semantic errors in transcoding tasks - the only errors she produced were orthographic or phonological-and she was quite good at reading and writing non-words to dictation, although she produced some lexicalizations. This suggests that EA was able to employ lexical as well as phonology-to-orthography and orthography-to-phonology conversion procedures when performing transcoding tasks.

The contrast in performance between these two patients prompted an investigation of the relationship between oral and written production and of the role of orthographic and phonological information in lexical selection. The two patients were asked to provide successive responses to a picture, alternating the modality of output: for example, in response to a picture, the patients could be asked to say its name, then to write it, then to say it again. In this task, WB produced a significant number of lexically inconsistent responses. For example, in response to the picture of a lion, WB would say "lion," write "tiger," and then say "lion" again. Lexically inconsistent responses generally involved semantically related items. Moreover, in trials in which his two first responses were lexically inconsistent, his third response was more often similar to the first responses (within modality consistency) than to the second response (across modality consistency). In contrast, EA's responses were virtually always lexically consistent: for example, in response to a picture of a comb, EA would say "comb," write "comb," and say "comb" again. These two patterns of response-often inconsistent across output modality for WB and never inconsistent for EA-were equally observed in the oral-written-oral and written-oral-written naming tasks.

The co-occurrence of a deficit in the orthography-to-phonology and phonologyto-orthography conversion procedures with the production of lexically inconsistent responses in successive naming tasks is in line with previous findings reported in the literature (Beaton et al., 1997; Miceli et al., 1997; Rapp et al., 1997). Patients WMA, $\mathrm{PW}$, and MGK often provided response sequences involving different lexical items. These patients also produced semantic errors in simple transcoding tasks such as 
reading or writing to dictation. ${ }^{2}$ All of them had damage to the lexico-semantic system and to the sub-lexical conversion processes (like WB). In contrast, patients PGE and GIM did not produce such inconsistent sequences, and did not produce semantic errors in transcoding tasks. Although these patients also suffered from a lexico-semantic deficit, they did not have damage to the sublexical conversion procedures (just like EA). Therefore the results reported above, combined with previous studies (see also Miceli et al., 1999), confirm that given a lexico-semantic deficit the occurrence of inconsistent responses - and of semantic errors in transcoding tasks - is closely tied to the availability of grapheme-to-phoneme (and vice versa) conversion rules in the system. As we will discuss below, these results have important implications for specific aspects of the process of activation and selection in models of lexical access.

The observation that inconsistent responses occur only with those patients with damage to the conversion procedures can be easily accommodated by assuming a "summation of activation" hypothesis (Hillis \& Caramazza, 1991, 1995; Patterson \& Hodges, 1992). According to this hypothesis, the activation of lexical entries occurs not only on the basis of semantic information extracted from visual processing of the picture but also - when available - on the basis of form-specific information provided by conversion procedures. Briefly stated, this hypothesis accounts for the consistency of EA's (and PGE's and GIM's) responses in the sequential naming task in the following manner. During the production of the first response (e.g., saying "comb"), the conversion procedures allow the activation of orthographic information corresponding to the pronounced word. When the second lexical selection (for writing) is carried out, the orthographic information just made available summates with the information produced by the new processing of the picture. In this way, the activation produced by the application of conversion procedures supports a re-use of the same lexical item in the second response of the trial. By contrast, in those patients where conversion procedures are not available - as is the case for WB-the production of the first response of a trial in a given modality (e.g., oral) does not lead to substantial activation of information in the other modality (e.g., orthographic information). Therefore, when the second response is produced, lexical selection is made solely on the basis of the information provided by the current processing of the picture. A deficit in the lexico-semantic system might then lead to a different lexical selection than the one provided in the previous response.

A somewhat similar impact of form-specific information on the process of lexical selection has been observed in other types of patients whose linguistic impairment does not primarily lead to the production of semantic errors, but rather to anomic states. A patient with this type of deficit will not produce any overt response in a significant number of trials of a picture-naming task. Because this naming difficulty is not necessarily accompanied by semantic impairment of the items involved, this deficit is often interpreted as arising at the level of lexical retrieval (Badecker, Miozzo, \& Zanuttini, 1995; Gainotti, Silveri, Villa, \& Miceli, 1986; Henaff-Gonon, Bruckert, \& Michel, 1989; Kay \& Ellis, 1987; Lambon Ralph, Sage, \& Roberts, 2000). A common finding among this type of patients is that phonological cues and miscues can have an impact on the outcome of a naming trial. In trials leading to an anomic state-for example if the patient is unable to produce a response in the presence of a picture of a tiger-providing him/her with a phonological cue (e.g., /t/) will significantly improve the likelihood of a correct response. What is more, miscueing the patient - e.g., by providing phonological information about a semantic

${ }^{2}$ PW produced very few semantic errors in reading. 
coordinate: /1/ of lion-can hamper performance and lead to the production of a semantic error ("lion;" Hillis \& Caramazza, 1995; Howard \& Orchard-Lisle, 1984; Lambon Ralph et al., 2000; see also Katz \& Lanzoni, 1997). Often these patients immediately reject these response as wrong, but only after producing them. The crucial point for our purposes then is that phonological information-the cue or miscue - can have an impact on the process of lexical selection, presumably by providing a little extra activation that will lead to the selection of a candidate already activated on the basis of semantic information.

There are at least two specific ways to implement computationally the impact of form-specific information on lexical selection: either directly between lexical forms or through sub-lexical representations. The first view postulates direct connections between lexical representations in the phonological and orthographic lexicons. The activation of a phonological lexical entry would produce the activation of the corresponding orthographic lexical entry, and vice versa (e.g., Allport \& Funnell, 1981; Patterson \& Shewell, 1987). The basic point of this assumption is that the mechanism of summation works on a word-to-word basis. Alternatively, the activation of a phonological lexical entry would produce the activation of sub-lexical (phoneme) representations. These would in turn activate orthographic sub-lexical representations through sub-lexical conversion procedures. Finally, the activation of these sublexical units would activate the corresponding lexical units (the same mechanism would also apply in the reverse direction; see for example Hillis \& Caramazza, 1991 or Patterson \& Hodges, 1992). Notice that this account requires postulating that activation can spread from the sublexical to the lexical units.

The data presented here do not allow us to distinguish between these two hypotheses, or between alternate processing accounts of the observations (see Hillis \& Caramazza, 1991, for a thorough discussion of this issue). What these results as well as other data reported in the literature strongly suggest however is that the availability of conversion procedures has an influence the process of lexical selection. In the following section we discuss the consequences of this conclusion for models of lexical access.

\subsection{Implementation of the summation hypothesis in models of lexical access}

The cross-talk between output lexicons is demonstrated here on the basis of the performance of two language-impaired patients. As we have seen, the effects we report are large and robust, suggesting that the summation processes must play an important role when they are available to these individuals. Obviously, in the case of normal unimpaired speakers, for whom lexical selection is in general conducted flawlessly, it is possible that the impact of such cross-talk on lexical selection is more limited. If one source of activation (e.g., semantic) allows by itself a correct, fast, and efficient retrieval of words, then the summation of activation from several sources might only produce minor effects. Still, the fact that such a cross-talk can have visible effects under certain circumstances indicates that the possibility of summation is a building block of the production system architecture. As such, it must be accounted for in general models of lexical access. In the remainder of this paper, we discuss this issue in detail by considering in turn various assumptions that can be made about the structure of the output lexicon.

Consider first models of language production where lexical selection is conducted over form-specific representations. In this type of model, the semantic system directly activates units in the phonological output lexicon when speaking, or in the orthographic output lexicon when writing (see Fig. 3A). Selection of an entry in either of these two modalities allows the retrieval of the properties of the word and its 
(A)

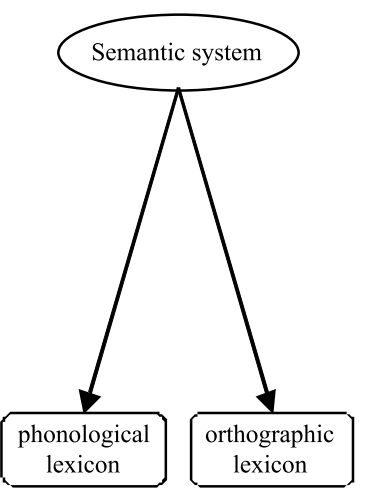

(B)

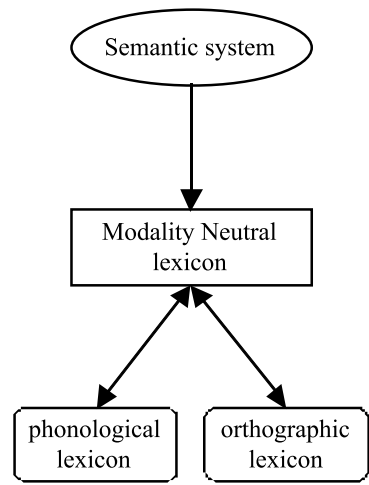

(C)

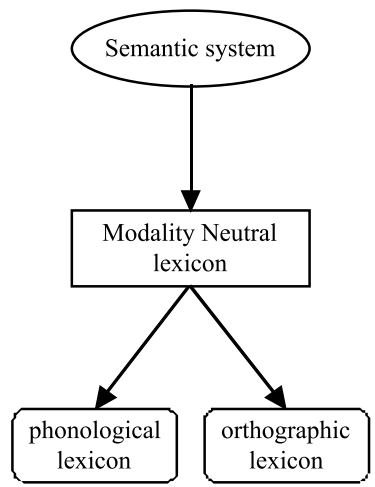

Fig. 3. Three possible assumptions about the implementation of lexical knowledge in a model of oral and written language production. (A) The semantic system directly activates the phonological and the orthographic output. In the example depicted activation is feed-forward only. (B) The semantic system activates a modality-neutral lexicon, which in turn activates the modality specific lexicons. Activation spreads in an interactive manner (from the modality-neutral representations to the output lexicons and vice versa). (C) The semantic system activates a modality-neutral lexicon, which in turn activates the modality specific lexicons. Activation spreads in a feed-forward fashion only.

subsequent production (Caramazza, 1997; Starreveld \& La Heij, 1996). Such a model would account for performance in a multiple picture-naming task in the following way. The first response of a sequence would for instance be selected over phonological lexical representations. The second response would then be selected over orthographic representations. Because these two lexical selections are conducted over different sets of representations, in the absence of any activation relationship between them-for instance, if conversion procedures are damaged-an error-prone lexico-semantic system may produce different responses in the two modalities. If, however, conversion procedures are available, then the selection of the second response would, to a certain degree, be constrained by what was produced in the first response. Activating and selecting a lexical item for oral output would lead to the activation of the corresponding orthographic lexical representation, favoring the use of the same (previously activated) word in the subsequent written response.

The main reason why models of this kind can account for the data reported in this paper is because lexical selection is conducted on form-specific lexicons, making plausible the assumption that lexical selection is potentially sensitive to the availability of form-specific information. Obviously, if a model of lexical access is based on a different set of assumptions, the summation hypothesis would need to be integrated in different ways. Consider the class of production models where semantics do not directly activate form-specific representations (e.g., Dell, 1986; Garrett, 1975; Levelt et al., 1999). In these models, lexical selection is conducted over a set of modality-neutral lexical representations - generally referred to as "lemmas"-that stand as mediators of the flow of activation between the semantic system and the phonological output lexicon. These lemmas specify the grammatical properties of the word they represent. Each of them is connected to a second lexical representation (the word's "lexeme") that encodes the phonological properties of the word (see Figs. 3B and C).

Most models implementing this hypothesis are based on research conducted in the field of speech production, and they remain silent on issues of written production. Because the summation hypothesis is based upon the interaction of phonological and 
orthographic information, it is not immediately obvious how it could be implemented in models of this kind. Still, it remains possible to evaluate how a theory would account for the phenomena reported in this study by extrapolating from the explicit assumptions made by some speech production models. In doing so we will postulate that the modality-neutral lexical representations (e.g., lemmas) used for speaking are also used to access the orthographic output lexicon during writing. In other words, in this working-model speaking would require the selection of a modality-neutral lexical representation and subsequently, the selection of the corresponding phonological lexeme ( $p$-lexeme); writing would require access to the same lemma representation and, in turn, the selection of the corresponding orthographic lexeme (o-lexeme) (see Figs. 3B and C). ${ }^{3}$

In models with modality-neutral lexical representations, a straightforward way to account for the occurrence of semantic errors would locate the noisy or faulty mechanism at the level directly activated by the semantic system: the modalityneutral lexical nodes. What needs to be explained then is the relationship we have observed between the availability (or lack thereof) of conversion procedures and the occurrence of lexically consistent and inconsistent responses-as well as the occurrence of semantic errors in transcoding tasks. In the following, the discussion of this issue is made on the basis of a secondary hypothesis about the spread of activation allowed in the system. We will consider first a model where information spreads in an interactive manner, and then a model where activation spreads in a feed-forward manner only.

If information is allowed to spread in an interactive manner between the modality-neutral lexical representations and the output lexicons, as soon as lexical representations receive activation, they start sending activation to their corresponding lexemes (Fig. 3B). This activation is allowed to feed back from the lexeme layer to the lexical layer, so that the information activated at the form level could have an impact on the process of lexical selection. ${ }^{4}$ Suppose the patient is asked to name the picture orally first: the activation of various modality-neutral lexical representations will in turn activate the corresponding $p$-lexemes. Note that because we have postulated that activation spreads through the system in a cascaded manner, it could be expected that $o$-lexemes are also activated. However, because the subject ends up producing an oral response, it must also be the case that the $p$-lexemes get a higher amount of activation in order for the system to know which output lexicon to

\footnotetext{
${ }^{3}$ In principle, it is also possible to extend these speech production models to writing by postulating different lemmas for oral and written production. This would mean that the process of lexical selection is conducted over different sets of representations. As a consequence, the interpretation of the role of conversion procedures in the process of lexical selection could be roughly parallel to that proposed for single layer models discussed previously. For this explanation to work, specific assumptions about the spread of activation should be made. In particular, it would require that form-specific information - that produced by the conversion procedures - affects the process of lexical selection. That is to say, for summation to occur, the levels of activation of form-specific lexemes must be allowed to have an impact on the activation of the modality-neutral representations (in relation to this point, see discussion of models of lexical access with interactive spread of activation, below).

${ }^{4}$ One of the original motivations for postulating such feedback in models of lexical access in speech production was the observation of phonological effects on lexical selection. For example, one (muchdebated) observation is that of so-called mixed errors in slips of the tongue produced by normal speakers or patients. A mixed error occurs when a speaker erroneously produces a word that is both semantically and phonologically related to the target word he/she intended to say (e.g., saying "dog" for "hog"). According to some authors, mixed errors occur more frequently than expected by chance in error corpora because activation flows back from the phonological layer to the modality-neutral layer (for discussion of this issue, see: Baars, 1980; Baars, Motley, \& McKay, 1975; Berg, 1986; Best, 1996; Blanken, 1998; Dell, 1986, 1988; Dell \& Reich, 1981; Gagnon, Schwartz, Martin, Dell, \& Saffran, 1997; Garrett, 1992; Stemberger, 1985).
} 
use. At some point in time, the modality-neutral lexical representation with the highest activation-possibly, but not necessarily, the correct response-will be selected (e.g., it will receive a boost of activation). Subsequently, the corresponding $p$ lexeme will be selected. If phonology-to-orthography conversion procedures were available, such extra activation would be transmitted to the corresponding $o$-lexeme in the orthographic output lexicon. This extra residual activation would have an impact on the lexical selection for the subsequent written response: it will favor the re-use of the same lexical item via feedback to the corresponding modality-neutral lexical representation. Under these conditions, lexically consistent responses are expected.

If, however, conversion procedures are not available, the selection of the $p$-lexeme should have no direct effect on the activation levels of $o$-lexemes. Therefore, although $o$-lexemes might have received some priming, the one corresponding to the word produced orally does not have a substantial advantage. In this scenario, the modality-neutral lexical representation that has just been selected will benefit from some residual activation, but it will only benefit from residual feedback from the $p$-lexeme. In other words, the only difference between the situation where conversion procedures are available and the situation where they are not is that in the first case both $p$ - and $o$-lexemes are supporting the activation of the modality-neutral representation whereas in the latter case this support comes only from the $p$-lexeme. The interactive working model would have to account for the reported results on the basis of this difference. One could for instance propose that lexical selection for writing is more likely to produce a response that is different from the response produced in the oral modality when only the $p$-lexeme produces feedback than when both the $p$-and the $o$-lexemes are actively producing feedback.

This type of explanation would seem to account for the observation that lexically inconsistent responses are only produced by patients suffering from a deficit of conversion procedures and not by patients in whom these procedures are available. Notice however that the robust and reliable contrast of performance that is observed between these two types of patients would be explained in terms of a subtle difference in levels of feedback affecting lexical selection. Because it is generally thought that the impact of feedback is small relative to that of direct activation, the viability of an actual quantitative implementation of this working model remains to be demonstrated. Therefore, although the interactive working-model cannot be rejected $a$ priori, its ability to account for the strict dissociation we report in a robust manner is not straightforward.

Consider now a model with a level of modality-neutral lexical representations that addresses both the phonological and the orthographic output, but where the activation spreads only in a feed-forward fashion (Fig. 3C). Under these assumptions lexical selection is carried out solely on the basis of activation stemming from the semantic system. This process should therefore be independent of the state of activation of the modality-specific lexicons and would seem at odds with the data reported in this paper. Can we modify this model in such a way that it keeps its core assumption but still accounts for the finding that the occurrence of lexically inconsistent responses in multiple picture-naming tasks is closely tied to the availability of conversion procedures?

One way to account for this finding is to modify the proposed interpretation of the mechanism responsible for the occurrence of semantic errors in these patients. It could be postulated that semantic errors are not due to difficulties in the process of lexical selection carried over modality-neutral representations but rather to difficulties in the retrieval of the $o$ - and $p$-lexemes. Such difficulties could for instance be due to problems in sending activation to the lexeme through the lexical node to 
lexeme link or to the impossibility of recovering the information represented at the lexeme level (see Caramazza, 1997; Caramazza \& Miozzo, 1998; Roelofs, Meyer, \& Levelt, 1998, for discussion of these possibilities). That is to say, lexical selection over modality-neutral representations would generally be conducted flawlessly in these patients, but a lexical error would arise as a failure to map a selected modalityneutral lexical representation onto its form-specific lexeme. ${ }^{5}$ For example, when naming the picture of a "lion," these patients would access the corresponding lemma correctly; however, because the $p$-lexeme may be temporarily unavailable, the system would not be able to produce the corresponding word. Following this temporary unavailability, the system would "reconsider" the lexical selection it just made and proceed by selecting a different candidate from among other highly activated items (e.g., "tiger"). If the $p$-lexeme of that second candidate is available for retrieval, articulation can proceed in the form of a semantic error. A similar reasoning would apply to written production.

On the basis of this set of assumptions, the occurrence of lexically inconsistent responses in the multiple picture-naming task could be explained as follows. When producing the first response, the system selects a modality-neutral lexical representation, retrieves the corresponding lexeme and produces it. If the $o$-lexeme of that word cannot be retrieved while processing the second response, the system will reconsider its lexical selection and, as suggested earlier, will select another lemma. A trial proceeding in this fashion-or in any of its straightforward variants-would yield lexically inconsistent responses across modalities. If the automatic conversion procedures between the phonological and the orthographic lexicons are available, the activation of a lexeme in one modality (e.g., phonological) will provide a boost of activation to the corresponding (e.g., orthographic) lexeme. This would reduce the likelihood that the $o$-lexeme would be unavailable when the second response has to be produced. Thus lexically inconsistent responses will not be expected. If the conversion procedures are not available, however, when a required $o$-lexeme cannot be retrieved the proposal of a reselection in the modality-neutral lexicon would be invoked and thereby account for the production of lexically inconsistent responses.

As can be seen, a model of speech and written production with modality-neutral lexical representations and a feed-forward propagation of activation could account for the data reported here (see also Rapp \& Caramazza, 2002). The interpretation of the deficit in terms of the unavailability of $p$ - and $o$-lexemes would allow an explanation of the occurrence of lexically inconsistent responses as the result of reselections at the level of the modality-neutral representations. However, despite its apparent explanatory power, one can raise both theoretical and empirical objections to this proposal. For example, some difficulties arise when making the central claim of reselection more explicit, as it is not obvious what could trigger it. One could postulate that the unavailability of information in the form-specific lexicon, beyond the modality-neutral representation, triggers re-selection. Note, however, that a mechanism of this sort would require that information stemming from the formspecific representation reaches the modality-neutral representations, in violation of the original assumption of feed-forward activation. Alternatively, it could be proposed that what triggers reselection is not directly the unavailability of form-specific information but rather another variable, such as time. The system would reselect in trials where the $p$-lexeme is unavailable after a pre-specified amount of time has elapsed without any overt production. Given the fact that selection is generally based

\footnotetext{
${ }^{5}$ In fact, it could be that the selection in the modality-neutral lexicon is also faulty in its own right. However, for simplicity, we will concentrate on the aspect of the deficit that is crucial for accounting for the reported pattern of results under the interpretation entertained here.
} 
on the relative levels of activation of candidates, for the second selection to converge on a different item the reselection mechanism should in some way inhibit or exclude the highly activated candidate selected in the first place. ${ }^{6}$ This move would save the feed-forward assumption but would require a number of sophisticated specifications for lexical selection. These specifications do not seem to be in line with current views about the mechanism of selection, and would need to be motivated independently.

The proposal of a reselection mechanism compensating for the temporary unavailability of form-specific information could be further constrained by considering other types of patients. We mentioned earlier the cases of patients who are unable to come up with the name of a picture in the face of relatively well preserved semantic and output systems (Badecker et al., 1995; Gainotti et al., 1986; Henaff-Gonon et al., 1989; Kay \& Ellis, 1987; Lambon Ralph et al., 2000). The performance of this type of patients has often been interpreted as the temporary unavailability of at least part of the form-specific information required for articulating the response (Badecker et al., 1995; Henaff-Gonon et al., 1989; consider also the case of normal subjects in tip-ofthe-tongue states: Caramazza \& Miozzo, 1997; Miozzo \& Caramazza, 1997; Vigliocco, Antonini, \& Garrett, 1997). In light of this interpretation, these cases provide grounds for a (cautious) comparison with the cases reported in the present paper. The reselection hypothesis states that in patients like WB lexical selection is reconsidered automatically when form-specific information is unavailable, thus ensuring that an answer is provided in the trial. Specifically, WB produces very few omissions-no responses-in naming tasks. In the different lexical tasks reported here, he produced no responses in $2.7 \%$ of the trials $(46 / 1722)$. If only picture naming is considered, the figure is $1.9 \%$ (10/520 trials). In the case of anomic states-as well as TOT states-the consequences of the unavailability of form-specific information seem to be different. In this population, the rate of omissions is very high, suggesting that lexical reselection is not systematically applied. If this line of reasoning were valid, then the account of the data on the basis of lexical reselection would have to explain why the unavailability of form-specific information can lead to such different outcomes.

It appears then that the working model with modality-neutral lexical representations and feed-forward activation encounters a number of difficulties in accounting for the data reported here. Its major problem stems from the fact that phonological and orthographic information-available when conversion procedures are operational-affects the form-specific lexical representations, while lexical selection operates at a modality independent level. This modality independent level is by hypothesis blind to the activation present in the form-specific lexicons. We have seen that this difficulty can be dealt with, but at the price of proposing complicated assumptions, for example, about the selection mechanism.

\section{Conclusion}

We have presented the cases of two patients-WB and EA-who suffer from a somewhat similar deficit in the lexico-semantic system, as evidenced by their production of semantic errors in picture-naming tasks. These patients differ in the extent to which they have access to phonology-to-orthography and orthography-to-phonology conversion procedures: these are very much impaired for WB and relatively

\footnotetext{
${ }^{6}$ If reselection implies inhibition of the lexical item that was first selected, it would not be clear either why the third response in a lexically inconsistent trial would be on average more similar to the first response than to the second response.
} 
well preserved for EA. In accordance with previous findings reported in the literature, we found that the unavailability of these conversion procedures co-occurs with the observation of semantic errors in transcoding tasks (such as reading and writing to dictation) and of lexically inconsistent responses in multiple picture-naming tasks. This pattern of results was interpreted as support for the "summation hypothesis" according to which various sources of information-notably semantic information and form-specific information-contribute to the process of lexical selection. We then discussed the integration of these observations in a model of lexical access in spoken and written language production. The results reported here could be easily integrated in models where phonology and orthography are directly involved in the process of lexical selection. This would be the case if this selection is carried out over formspecific representations. Our discussion also showed that our results stand as a challenge for the general class of oral and written production models where lexical selection is conducted over a modality-neutral lexicon independent of form-specificphonological or orthographic_information.

\section{References}

Allport, D. A., \& Funnell, E. (1981). Components of the mental lexicon. Philosophical Transactions of the Royal Society of London, 295, 397-410.

Baars, B. J. (1980). On eliciting predictable speech errors in the laboratory. In V. A. Fromkin (Ed.), Errors in linguistic performance: Slips of the tongue, ear, pen, and hand (pp. 307-318). New York: Academic Press.

Baars, B. J., Motley, J. T., \& McKay, D. (1975). Output editing for lexical status from artificially elicited slips of the tongue. Journal of Verbal learning and Verbal Behavior, 14, 382-391.

Badecker, W., Miozzo, M., \& Zanuttini, R. (1995). The two-stage model of lexical retrieval: Evidence from a case of anomia with selective preservation of grammatical gender. Cognition, 57, 193-216.

Beaton, A., Guest, J., \& Ved, R. (1997). Semantic errors of naming, reading, writing, and drawing following left-hemisphere infarction. Cognitive Neuropsychology, 14, 459-478.

Berg, T. (1986). The problems of language control: Editing, monitoring, and feedback. Psychological Research, 48, 133-144.

Best, W. (1996). When racquest are baskets but basekts are bisquits, where do the words come from? A single case study of formal paraphasic errors in aphasia. Cognitive Neuropsychology, 13, 443-480.

Blanken, G. (1998). Lexicalisation in speech production: Evidence from form-related word substitutions in aphasia. Cognitive Neuropsychology, 15(4), 321-360.

Bub, D., Cancelliere, A., \& Kertesz, A. (1985). Whole-word and analytic translation of spelling-to-sound in a non-semantic reader. In K. E. Patterson, J. C. Marshall, \& M. Coltheart (Eds.), Surface dyslexia. London: Erlbaum.

Caramazza, A. (1997). How many levels of processing are there in lexical access? Cognitive Neuropsychology, 14, 177-208.

Caramazza, A., \& Hillis, A. E. (1990). Where do semantic errors come from. Cortex, 26, 95-122.

Caramazza, A., \& Miozzo, M. (1997). The relation between syntactic and phonological knowledge in lexical access: Evidence from the tip-of-the-tongue phenomenon. Cognition, 64, 309-343.

Caramazza, A., \& Miozzo, M. (1998). More is not always better: A response to Roelofs, Meyer, and Levelt. Cognition, 69, 231-241.

Coslett, H. B. (1991). Read but not write idea: Evidence for a third reading mechanism. Brain and Language, 40, 425-443.

Dell, G. S. (1986). A spreading activation theory of retrieval in sentence production. Psychological Review, 93, 283-321.

Dell, G. S. (1988). The retrieval of phonological forms in production: Test of predictions from a connectionist model. Journal of Memory and Language, 27, 124-142.

Dell, G. S., \& Reich, P. A. (1981). Stages in sentence production: An analysis of speech error data. Journal of Verbal Learning and Verbal Behavior, 20, 611-629.

Funnell, E. (1983). Phonological processes in reading: New evidence from acquired dyslexia. British Journal of Psychology, 74, 159-180.

Gagnon, D. A., Schwartz, M. F., Martin, N., Dell, G. S., \& Saffran, E. M. (1997). The origins of formal paraphasias in aphasics' picture naming. Brain and Language, 59(3), 450-472. 
Gainotti, G., Silveri, M., Villa, G., \& Miceli, G. (1986). Anomia with and without lexical comprehension disorders. Brain and Language, 29, 18-33.

Garrett, M. F. (1975). The analysis of sentence production. In G. Bower (Ed.), Psychology of learning and motivation (Vol. 9). New York: Academic Press.

Garrett, M. F. (1992). Disorders of lexical selection. Cognition, 42, 143-180.

Geschwind, N. (1969). Problems in the anatomical understanding of aphasia. In A. L. Benton (Ed.), Contributions of clinical neuropsychology. Chicago, IL: University of Chicago Press.

Goodglass, H., Kaplan, E., \& Barresi, B. (2001). The Boston diagnostic aphasia examination (3rd ed). Baltimore, MD: Lippincott Williams \& Wilkins.

Grashey, H. (1885). On aphasia and its relations to percetion (Über Aphasie und ihre Beziehungen zur Wahrnehmung). Archiv für Psychiatrie une Nervenkrankheiten, 16, 654-688.

Head, H. (1926). Aphasia and kindred disorders of speech. London: Cambridge University Press.

Hecaen, H., \& Angelergues, R. (1965). Pathologie du langage (Vol. 1). Paris: Larousse.

Henaff-Gonon, M., Bruckert, R., \& Michel, F. (1989). Lexicalization in an anomic patient. Neuropsychologia, 27, 391-407.

Hillis, A. E., \& Caramazza, A. (1991). Mechanisms for accessing lexical representations for output: Evidence from a category-specific semantic deficit. Brain and Language, 40, 106-144.

Hillis, A. E., \& Caramazza, A. (1995). Converging evidence for the interaction of semantic and sublexical phonological information in accessing lexical representation for spoken output. Cognitive Neuropsychology, 12, 187-227.

Howard, D., \& Orchard-Lisle, V. (1984). On the origin of semantic errors in naming: Evidence from a case of a global aphasic. Cognitive Neuropsychology, 1, 163-190.

Howard, D., \& Patterson, K. (1992). Pyramids and palmtrees: A test of semantic access from words and pictures. Bury St Edmunds, Suffolk: Thames Valley Test Company.

Katz, R. B., \& Lanzoni, S. M. (1997). Activation of the phonological lexicon for reading and object naming in deep dyslexia. Brain and Language, 58(1), 46-60.

Kay, J., \& Ellis, E. W. (1987). A cognitive neuropsychological case study of anomia: Implications for psychological models of word retrieval. Brain, 110, 613-629.

Lambon Ralph, M. A., Sage, K., \& Roberts, J. (2000). Classical anomia: A neuropsychological perspective on speech production. Neuropsychologia, 38, 186-202.

Levelt, W. J. M., Roelofs, A., \& Meyer, A. (1999). A theory of lexical access in speech production. Behavioral and Brain Sciences, 22, 1-75.

Lhermitte, F., \& Dérouesné, J. (1974). Paraphasies et jargonaphasie dans le langage oral avec conservation du langage écrit. Revue Neurologique, 130, 21-38.

Lichtheim, L. (1885). On aphasia (Über Aphasie). Deutches Archiv für klinische Medizin, 36, 204 268.

Luria, A. R. (1966). Higher cortical functions in man. New York: Basic Books.

Miceli, G., Benvegnù, B., Capasso, R., \& Caramazza, A. (1997). The independence of phonological and orhtographic lexical forms: Evidence from aphasia. Cognitive Neuropsychology, 14, 35-69.

Miceli, G., \& Capasso, R. (1997). Semantic errors as neuropsychological evidence for the independence and the interaction of orthographic and phonological word forms. Language and Cognitive Processes, $12,733-764$

Miceli, G., Capasso, R., \& Caramazza, A. (1999). Sublexical conversion procedures and the interaction of phonological and orthographic lexical forms. Cognitive Neuropsychology, 16, 557-572.

Miozzo, M., \& Caramazza, A. (1997). The retrieval of lexical-syntactic features in tip-of-the-tongue states. Journal of Experimental Psychology: Learning, Memory, and Cognition, 23, 1410-1423.

Nickels, L. (1997). Spoken word production and its breakdown in aphasia. Hove, UK: Psychology Press.

Nickels, L. (2001). Spoken word production. In B. Rapp (Ed.), The handbook of cognitive neuropsychology: What deficits reveal about the human mind (pp. 291-320). Philadelphia, PA, US: Psychology Press/ Taylor \& Francis.

Patterson, K., \& Hodges, J. R. (1992). Deterioration of word meaning: Implications for reading. Neuropsychologia, 30, 1025-1040.

Patterson, K., \& Shewell, C. (1987). Speak and spell: Dissociations and word-class effects. In M. Coltheart, G. Sartori, \& R. Job (Eds.), The cognitive neuropsychology of language. London: Erlbaum.

Rapp, B., Benzig, L., \& Caramazza, A. (1997). The autonomy of lexical orthography. Cognitive Neuropsychology, 14, 71-104.

Rapp, B., \& Caramazza, A. (2002). Selective difficulties with spoken nouns and written verbs: A single case study. Journal of Neurolinguistics, 15, 373-402.

Rapp, B., Epstein, C., \& Tainturier, M. -J. (in press). The integration of information across lexical and sublexical processes in spelling. Cognitive Neuropsychology, 19(1), 1-29.

Riddoch, M. J., \& Humphreys, G. W. (1992). The Birmingham object recognition battery (BORB). Hove, UK: Erlbaum. 
Roelofs, A., Meyer, A. S., \& Levelt, W. J. M. (1998). A case for the lemma/lexeme distinction in models of speaking: Comment on Caramazza and Miozzo (1997). Cognition, 69, 219-230.

Sartori, G., Masterson, J., \& Job, R. (1987). Direct route reading and the locus of lexical decision. In M. Coltheart, G. Sartori, \& R. Job (Eds.), Cognitive neuropsychology of language. London: Erlbaum.

Schwartz, M. F., Saffran, E. M., \& Marin, O. S. M. (1980). Fractionating the reading process in dementia: Evidence for word-specific print-to-sound associations. In M. Coltheart, K. E. Patterson, \& J. C. Marshall (Eds.), Deep dyslexia. London: Routledge \& Kegan Paul.

Semenza, C., Cipolitti, L., \& Denes, G. (1992). Reading aloud in jargonaphasia: An unusual dissociation in speech output. Journal of Neurology, Neurosurgery, and Psychiatry, 55, 205-208.

Snodgrass, J. G., \& Vanderwart, M. (1980). A standardized set of 260 pictures: Norms for name agreement, image agreement, familiarity, and visual complexity. Journal of Experimental Psychology: Human Learning and Memory, 6, 174-215.

Starreveld, P. A., \& La Heij, W. (1996). Time-course analysis of semantic and orthographic context effects in picture naming. Journal of Experimental Psychology: Learning, Memory, and Cognition, 22, 896-918.

Stemberger, J. P. (1985). An interactive activation model of language production. In A. W. Ellis (Ed.), Progress in the psychology of language (Vol. 1, pp. 143-186). Hillsdale, NJ: Erlbaum.

Vigliocco, G., Antonini, T., \& Garrett, M. F. (1997). Grammatical gender is on the tip of Italian tongues. Psychological Science, 8, 314-317. 